\title{
Magnetism in iron as a function of pressure
}

\author{
Gerd Steinle-Neumann $^{1,4}$, R E Cohen ${ }^{2}$ and Lars Stixrude ${ }^{3}$ \\ ${ }^{1}$ Bayerisches Geoinstitut, University Bayreuth, 95440 Bayreuth, Germany \\ 2 Geophysical Laboratory, Carnegie Institution of Washington, Washington, DC 20015, USA \\ ${ }^{3}$ Geological Sciences, University of Michigan, Ann Arbor, MI 48104, USA \\ E-mail: g.steinle-neumann@uni-bayreuth.de
}

Received 20 January 2004

Published 26 March 2004

Online at stacks.iop.org/JPhysCM/16/S1109

DOI: $10.1088 / 0953-8984 / 16 / 14 / 020$

\begin{abstract}
Magnetism in iron plays a central role in understanding the physical properties of its polymorphs, including the close-packed high pressure phases. We explore the rich and complex magnetic structures of these phases in two ways. We use a first-principles based, magnetic tight-binding total energy model to study non-collinear magnetic structures, and an all-electron method to study the collinear state in hcp iron that we predict in the hcp iron stability range. For the non-collinear study we compute the magnetization energy and moments for various non-collinear ordered spin configurations. For fcc iron we find non-collinear structures with a wavevector $(0,0, q)$ with $q$ close to 0.5 to be energetically stable, in agreement with previous first-principles calculations. In the high pressure stability field of hcp iron we find a stable collinear antiferromagnetic structure (afmII), previously predicted with an allelectron method. We further investigate the afmII structure, computing physical properties from first principles that support the notion of antiferromagnetic correlations in hcp iron. We show that a recently observed anomalous splitting in Raman spectra of hep iron under compression can be quantitatively explained by spin-phonon interactions. To address the absence of Mössbauer splitting in experiments on hcp iron we have also calculated the hyperfine field of afmII iron and find it to be so small that the predicted splitting would be smaller than the resolution limit of experiments.
\end{abstract}

\section{Introduction}

Magnetism plays an important role in understanding the phase equilibria and physical properties of the iron polymorphs. The phase at ambient conditions, body centred cubic (bcc), is stabilized by the presence of ferromagnetic moments, as has been shown from density

4 Author to whom any correspondence should be addressed. 
Table 1. Comparison of the equation of state for hcp iron. Parameters are the zero pressure volume $\left(V_{0}\right)$, zero pressure bulk modulus $\left(K_{0}\right)$, and its pressure derivatives $K_{0}^{\prime}$. Experimental data are from [45], and the LAPW results are from [10].

\begin{tabular}{llll}
\hline hcp iron (GGA) & $V_{0}\left(\mathrm{bohr}^{3}\right)$ & $K_{0}(\mathrm{GPa})$ & $K_{0}^{\prime}$ \\
\hline Experiment & 75.4 & 165 & 5.3 \\
LAPW non-magnetic & 69.0 & 292 & 4.4 \\
LAPW afmII & 71.2 & 209 & 5.2 \\
TB non-magnetic & 68.8 & 297 & 4.6 \\
TB afmII & 70.4 & 213 & 6.1 \\
TB non-collinear & 70.5 & 203 & 6.3 \\
\hline
\end{tabular}

functional theory (DFT) based computations [1]. The high temperature phase, cubic close packed (fcc), has no ordered magnetic structure in its stability field, but magnetic correlations are responsible for the anti-Invar effect (large thermal expansivity) in this phase [2] and anomalous phonon dispersion [3].

Pressure has a large effect on the magnetic structure through the delicate balance between potential and kinetic energy that governs magnetism [4], resulting in a decrease of magnetic moments under compression, making the question of magnetism in iron under pressure of general interest. As magnetism has a strong influence on material properties, the magnetic structure of the high pressure phase of iron, hexagonal close packed (hcp), is of special interest in high pressure material research, for the study of impact phenomena in iron and steel [5] and for geophysics, as Earth's inner core is most likely composed of hcp iron [6]. Hcp iron is not quenchable to zero pressure, so magnetism must be studied in situ at high pressure. The absence of observable splitting in in situ Mössbauer experiments to low temperatures [7-9] places an upper limit of the hyperfine field (HFF) of only $5 \mathrm{kG}$ [9], leading to the conclusion that no significant magnetic moments are present. However, using collinear magnetic DFT based methods a stable antiferromagnetic (afm) state of hcp iron has been predicted [10], a finding that is robust within DFT computations using both local density (LDA) and generalized gradient approximation (GGA) to the exchange-correlation potentials [10-12], as well as magnetic tight-binding models with a Stoner-like exchange energy [13]. This structure (afmII, figure 1) is characterized by alternating spin up and down planes perpendicular to one of the basal planes in the hcp cell. The afmII phase has been predicted to be stable up to $60 \mathrm{GPa}$, and taking the afmII structure into account improves the agreement of the computed and experimental equation of state for hcp iron significantly (figure 2 and table 1) [10].

Recent experimental observations, including anomalous splitting of the Raman mode in hcp iron $[14,15]$, focus attention on this apparent discrepancy between theory and experiment. The Raman active phonon mode in a monatomic hcp system is the doubly degenerate transverse optical (TO) mode. Consequently, only one peak is expected in Raman spectra of hcp iron. However, experiments using low fluorescence synthetic diamonds show two peaks up to pressures of $40 \mathrm{GPa}[14,15]$ suggesting a symmetry lower than the atomic arrangement determined to be hcp by in situ X-ray diffraction [16].

When magnetic moments are collinear, electrons can be considered to be 'spin-up' or 'spin-down' in a global sense, i.e. there is a global magnetic quantization direction. This means that one can solve for the spin-up and spin-down electrons separately, and then combine the results to compute the total charge density. When moments are oblique to each other, the spin state is termed 'non-collinear'. In this case the problem does not factorize, and one must diagonalize a Hamiltonian of twice the order of the collinear system. A system can be noncollinear either in response to chemical or thermal disorder, or in order to minimize frustration. 


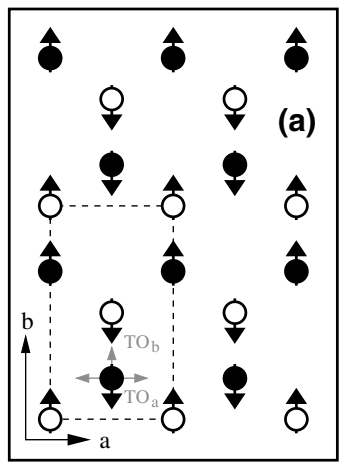

afmII (Pmma)

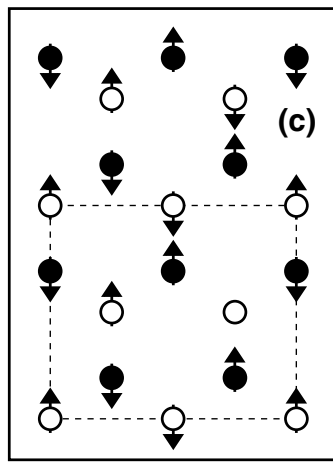

$\operatorname{ncl}(\alpha=0)=\operatorname{afmIII}(\mathrm{Pmmn})$

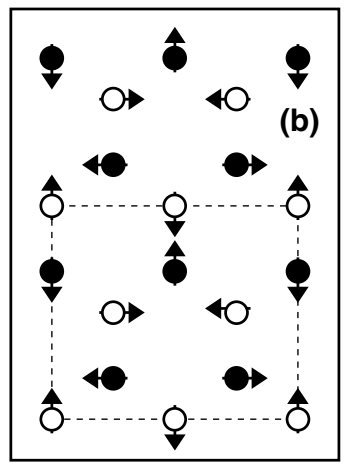

$\operatorname{ncl}\left(\operatorname{Pmn} 2{ }_{1}\right)$

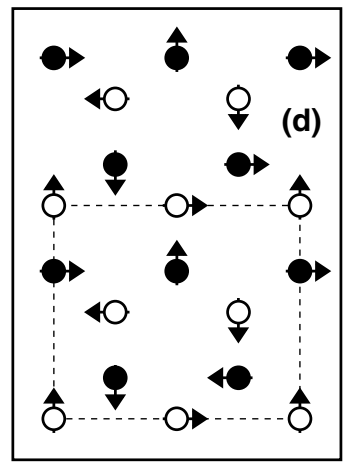

ncl4 (Pmn2 1$)$

Figure 1. Some of the magnetic structures for hep iron considered. Atoms at $z=1 / 4$ are shown with open symbols, and for $z=3 / 4$ with filled symbols; the orthorhombic unit cells of the structures are outlined (dashed lines), with the two planar basis vectors $a$ and $b$ given in (a). The arrows indicate spin-up and spin-down atoms. In (a) the previously found collinear state (afmII) is shown. Also indicated (in grey) are the two eigendirections for the transverse optical phonon modes. In (b) the non-collinear structure ncl (for $\alpha=90$ ) is shown. For $\alpha=0$ another collinear structure is obtained as shown in (c). A different type of non-collinear structure is shown in (d).

Both hcp and fcc lattices are frustrated with respect to antiferromagnetism, in that one cannot order these lattices antiferromagnetically so that all neighbours have opposite spins. This is known to lead to spin-waves and non-collinear magnetism in fcc iron, for which it is now understood that the magnetic ground state is a incommensurate spin-density wave, both from DFT calculations [17, 18] and studies of fcc iron precipitates [19].

Here we further explore magnetism in hep iron in two ways. First, we go beyond the collinear antiferromagnetic state and self-consistently relax the magnetic structures using a non-empirical tight-binding model with a Stoner-like exchange energy $[13,20]$. Second, we further explore the consequences of afm correlations by studying the influence of the afmII structure on physical observables. Using all-electron DFT we compute the phonon frequencies of the two TO phonon modes in afmII and compare them to the experimental measurements of the splitting in the Raman experiment. We also calculate the HFF to address the Mössbauer experiments. The use of the afmII structure in this context is motivated for two reasons. First, in the stability field of hcp iron (above $13 \mathrm{GPa}$ ) the non-collinear study did not yield a more stable structure than afmII. Second, the collinear afmII structure corresponds to a collective mode excitation of afm correlations on the hcp lattice, predicted to be stable at some finite temperature for the nearest-neighbour Heisenberg model of antiferromagnetism [21]. 

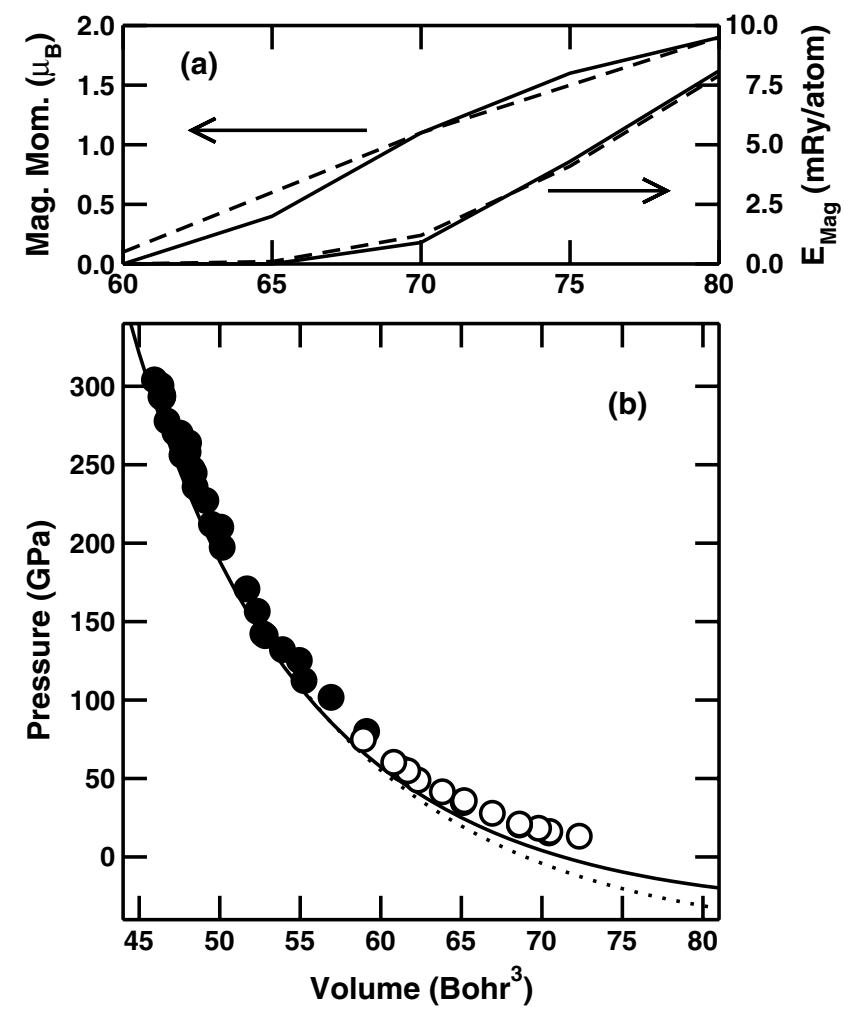

Figure 2. The equation of state for hcp iron. Panel (a) shows the magnetic moments (left-hand set of curves) and magnetization energies (right-hand set of curves) for the ncl ( $\alpha=90)$ (solid curves) and afmII (dashed curves) from the tight-binding calculations. Panel (b) compares experimental measurements of the equation of state from [16] and [45] with results for non-magnetic (dotted curve) and the antiferromagnetic calculations (solid curve). The afmII and ncl equations of state are identical on the scale of the figure.

\section{Method}

To study non-collinear magnetism in Fe, a first-principles based non-magnetic tight-binding model [22] is combined with a model for magnetism [23] that is described in detail elsewhere [13]. Similar models have been used previously for incorporating magnetism within a tight-binding approach [20, 23-26], but a first-principles tight-binding model has previously not been developed that includes constraining fields or that properly treats non-orthogonal tight-binding.

The model differs from the conventional Stoner model [27], in which a ferromagnetic instability is predicted by the inequality $I N(0)>1$, where $N(0)$ is the non-magnetic density of states at the Fermi level, and the extended Stoner model [28], in which $N$ is replaced by the effective density of states $\tilde{N}(M)=M / \delta \epsilon$, where $\delta \epsilon$ is the exchange splitting, in that our model allows for different hybridization depending on the magnetic state, accounting thereby for the actual magnetic structure, i.e. whether the system will be ferromagnetic, antiferromagnetic, or non-collinear.

We explore the influence of the afmII ordering on physical properties over the compression range where finite moments are predicted ( $\left.>60 \mathrm{bohr}^{3} / \mathrm{atom}\right)$ by means of the spinpolarized fully relativistic all-electron linearized-augmented plane-wave method (LAPW) with 
GGA [29]. We treat 3s, 3p, 3d, 4s, and $4 p$ states as valence electrons for all volumes and use $R_{\mathrm{MT}}=2.0$ bohr for the muffin tin radii, $R_{\mathrm{MT}} K_{\max }=9.0$, a $12 \times 6 \times 12$ special $k$-point mesh, and a temperature broadening of $5 \mathrm{mRyd}$. s- and p-states are treated with local orbitals. Tests with higher $k$-point sampling and smaller temperature broadening show that our results are well converged in terms of magnetic moments (within $0.05 \mu_{\mathrm{B}}$ ) and magnetization energies ( $0.02 \mathrm{mRyd} /$ atom $)$. We calculate the TO mode frequencies by the frozen phonon method: energy changes are evaluated in response to small displacements along the phonon eigenvector, with the second-order term yielding the frequency. The HFF is computed self-consistently from the spin-up and spin-down charge density at the nucleus averaged about the Thompson sphere, generalizing the Fermi contact interaction to the relativistic case [30]. The use of GGA is of critical importance for the magnetization energies in iron $[1,31]$ as well as for the HFF in the 3d transition metals [32].

To efficiently perform the calculations we fix the free parameter in the hcp structure, the axial ratio, to $c / a=1.6$, close to the experimentally [16] and computationally [10] determined equilibrium value. Tests showed that $c / a$ does not vary significantly $(<0.005)$. For the tightbinding calculations on eight-atom orthorhombic cells for hexagonal symmetry (see below) a $k$-point mesh of $12 \times 12 \times 12$ is used. For the eight-atom tetragonal supercells of the fcc structure, dense $12 \times 12 \times 4 k$-points are used.

\section{Results}

\subsection{Non-collinear studies}

The method employed in the study of non-collinear magnetism yields results that are in good agreement with previous self-consistent calculations for bcc iron, especially when a volumedependent Stoner parameter $I$ is used [20]. The lowest energy state for $\alpha$-Fe for the model at ambient and at higher pressures is ferromagnetic, in agreement with the first-principles LAPW self-consistent calculations [31].

Spin states in fcc iron have been studied extensively (for a review, see [18]), showing a great richness in non-collinear magnetic structures on the frustrated cubic lattice as the volume is varied. Here we determine the energies and moments for fcc iron with spiral spins along (001) and $\theta=\pi / 2$ using eight-atom supercells, allowing calculations for $(00 q)$ with $q=0, \pi / 4, \pi / 2,3 \pi / 4$, and $\pi$. The complex behaviour of magnetism with increasing pressures and varying spiral spin density wave states is qualitatively reproduced by the model (figure 3), compared with self-consistent calculations [17, 18, 33]. Quantitatively the results are sensitive to the value of $I$. In any case, there is some variation in self-consistent results for fcc iron, due to the extreme sensitivity of the magnetic structure to the basis set, $k$-point sampling, etc, as a result of the small energy scale. Furthermore, the magnetic ground state in fcc iron is sensitive to the atomic moment approximation [33] in the tight-binding model. In a spin density wave the moment is rather a field that varies with position in the crystal, and is not constant on each atom. Within the atomic moment approximation our results are reasonably consistent with the self-consistent results.

Like the fcc structure, the hcp lattice is geometrically frustrated in that it is not possible to have perfect collinear antiferromagnetic order on it: in the afmII structure, for example, each atom has four like spin (ferromagnetic) and eight unlike spin (afm) nearest neighbours (figure 1), maximizing the antiferromagnetic order. In the case of a nearest-neighbour (nn) Heisenberg model with energy $E=J_{1} \sum_{\mathrm{nn}} \vec{m}_{i} \cdot \vec{m}_{j}$, the energy is independent of the angle between the moments of one antiferromagnetic pair and another (figure 4) [21]. If non$\mathrm{nn}$ interactions are important, or if the Heisenberg model does not completely describe the 

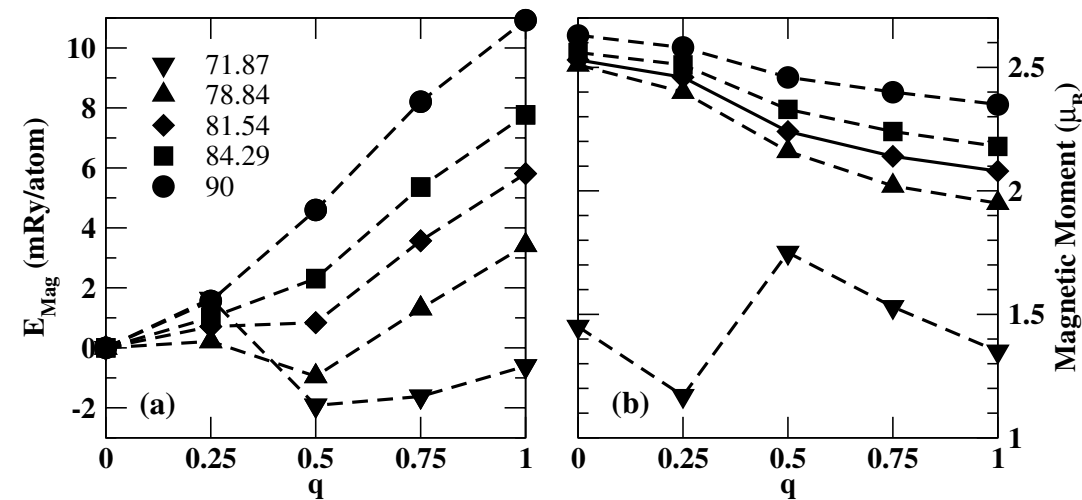

Figure 3. Variation of magnetization energy (a) and magnetic moments (b) versus wavevector $(0,0, q)$ for fcc iron with $q$ in units of $2 \pi / a$, where $a$ is the lattice constant. Lines for various unit cell volumes are given (see the legend in (a)). Wavevectors $q=0$ and 1 correspond to ferromagnetic and antiferromagnetic structures. $q=0.5$ is a non-collinear structure with an angle of $\pi / 2$ between neighbouring spins.

energetics, the energy might be further lowered if the afm pairs are oblique or perpendicular to each other (i.e. $\alpha \neq 0$ ). The energy does depend on which pairs are chosen to be antiferromagnetic (i.e. how the tetrahedra of figure 4 are connected), giving rise to different possible magnetic structures (figure 1). The collinear afmII structure has four atoms per unit cell in space group Pmma [10]. If we tile the lattice with the pattern shown in figure 4, cells with eight atoms are obtained (figure 1); there the collinear state has the space group Pmmn $(\alpha=0)$.

The energy of the ncl structure changes as a function of the angle $\alpha$ for different volumes for the ncl structure (figure 5). In all cases the energy decreases when $\alpha$ is varied from zero. Nevertheless, for volumes less than or equal to about $70 \mathrm{bohr}^{3}(\sim 2 \mathrm{GPa})$, the afmII structure is more stable than the ncl structure for any angle; therefore the afmII structure remains the

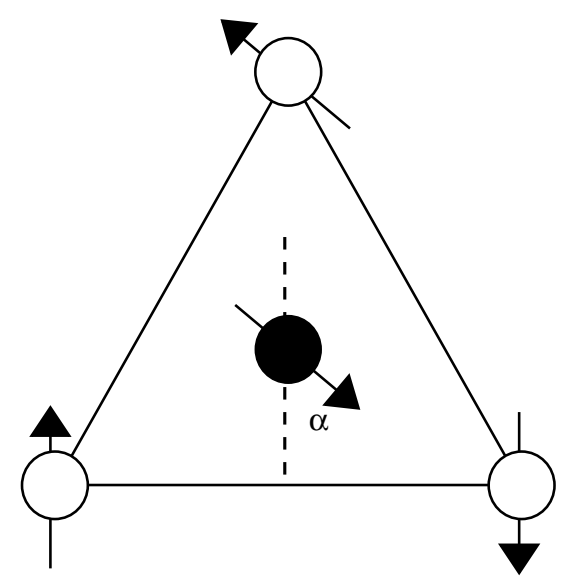

Figure 4. The motif for antiferromagnetic interactions on the hcp lattice (tetrahedron representation with open symbols at $z=1 / 4$ and filled symbol at $z=3 / 4$ ). One pair of antiferromagnetic iron atoms is at an angle $\alpha$ to the other. Collinear structures with space group symmetry Pmma (afmII) and Pmmn (afmIII) are represented by $\alpha=0$. See also figure 1 . 


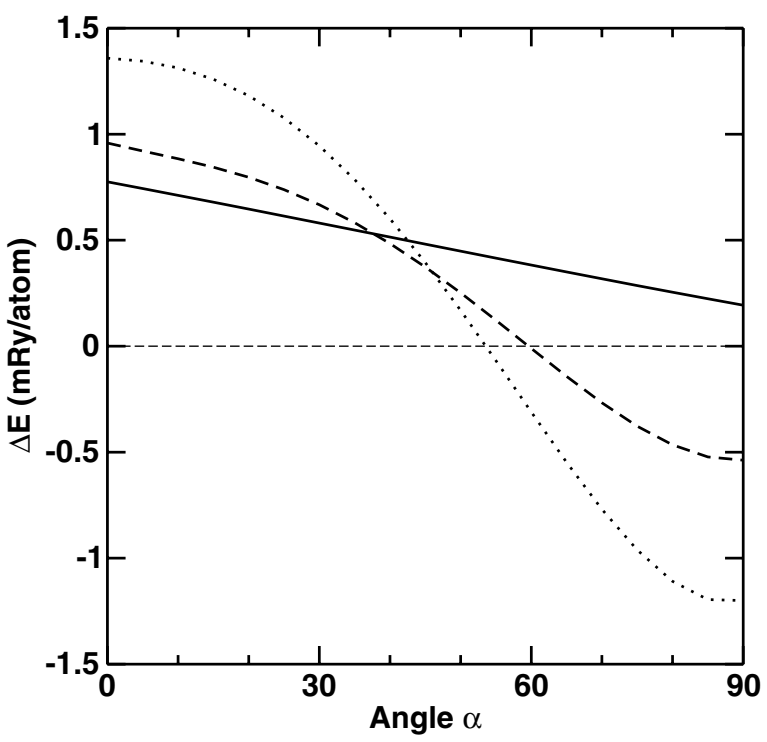

Figure 5. Energy as a function of angle $\alpha$ (see figure 4). The energy is given relative to the afmII structure (zero line), so that negative values show structures that are energetically favoured over afmII. The dotted, dashed, and solid lines are curves for volume/atom of 80, 75, and $70 \mathrm{bohr}^{3}$.

lowest energy structure we were able to find in the stability range of hcp iron, above $13 \mathrm{GPa}$. The equation of state of hcp iron changes little from afmII to ncl with $\alpha=90^{\circ}$ (figure 2 and table 1).

\subsection{Collinear structure}

Aside from being the lowest energy state, the afmII structure is also predicted to become stable at finite temperature in the nn Heisenberg afm model discussed above, through collective mode excitations [21], an entropic effect known as ordering by disorder [34], adding a second motivation to consider the afmII structure.

The Raman active TO phonon modes in the afmII structure are characterized by displacements of the close-packed planes with respect to each other, with the eigenvectors for the two modes along the orthorhombic axes (figure 1). Inspection of the structure reveals that fundamentally different spin interactions are involved in the two modes (figure 1). For displacements along the orthorhombic $a$-axis $\left(\mathrm{TO}_{a}\right)$, atoms approach nearest neighbours with unlike spin, corresponding to afm correlations. For displacements along the orthorhombic $b$-axis $\left(\mathrm{TO}_{b}\right)$, atoms alternately move towards or away from a nearest neighbour with like spin, corresponding to ferromagnetic correlations. The resulting potentials reflect the symmetry of the interaction: while for the $\mathrm{TO}_{b}$ mode we see considerable anharmonicity, there is none for the $\mathrm{TO}_{a}$ and the non-magnetic TO modes. We find that the $\mathrm{TO}_{a}$ mode frequency agrees well with that of the lower frequency, higher amplitude peak found in the Raman experiments [14, 15], and that the $\mathrm{TO}_{b}$ mode frequency corresponds to the experimentally observed satellite peak at higher frequency (figure 6). The magnitude of the predicted TO mode splitting decreases as the afm moment is reduced by compression, in excellent agreement with the observation in the Raman experiments (figure 6). The systematic offset of the calculated frequencies by approximately $20 \mathrm{~cm}^{-1}(<10 \%)$ is typical for a comparison of computed and measured phonon frequencies [35]. 

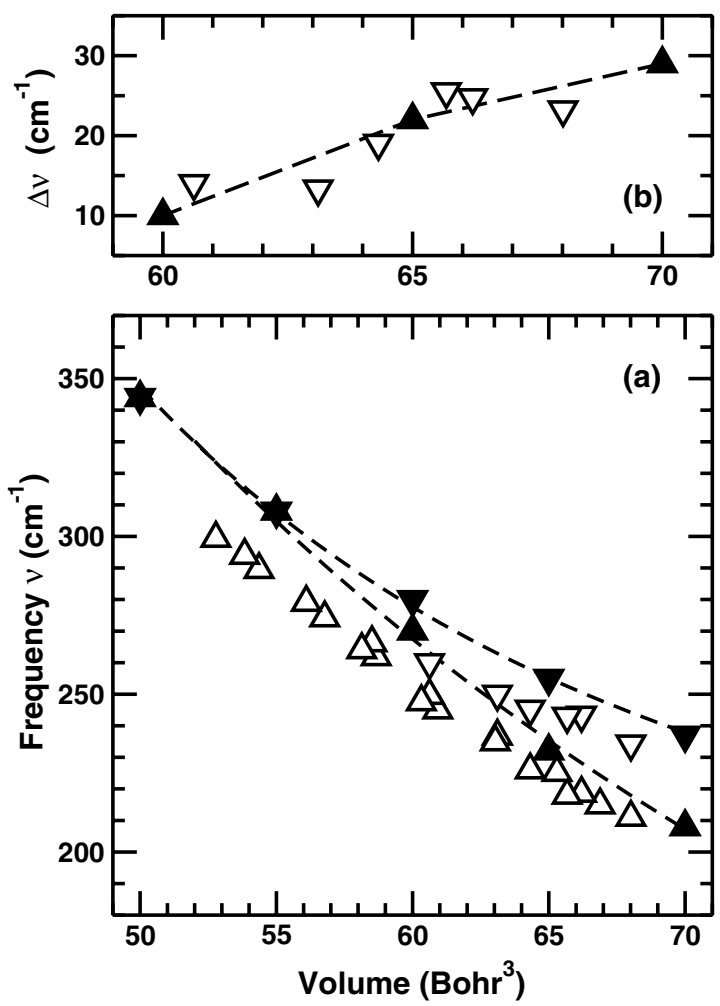

Figure 6. Transverse optical phonon modes for hexagonal iron. Panel (a) shows calculated frequencies (filled symbols) for $\mathrm{TO}_{a}$ (triangles up) and $\mathrm{TO}_{a}$ (triangles down) in the afmII structure in comparison to the Raman measurements $[14,15]$ (open symbols). Here the main peak (triangles up) and satellite peaks (triangles down) are distinguished. The dashed curves are polynomial fits in $V^{-2 / 3}$ to the results. Panel (b) compares the difference between $\mathrm{TO}_{b}$ and $\mathrm{TO}_{a}$ (filled symbols; the line is to guide the eye) and the experimental main and satellite peaks.

To address the Mössbauer experiments we have calculated the HFF for afmII iron, and find it to be only a few $\mathrm{kG}$ over its stability field (table 2). This is two orders of magnitude smaller than the HFF for fm bcc iron. For bcc iron we find $323 \mathrm{kG}$ at an atomic volume $\left(V=80\right.$ bohr $\left.^{3}\right)$ close to the experimental zero pressure volume, in good agreement with the experimental saturation value $(339 \mathrm{kG})$, and obtain a slight decrease of the $\mathrm{HHF}$ as a function of compression (table 2). The separation of the outermost peaks in the Mössbauer spectrum (L1 and L6) is diagnostic of the HFF, and a typical value of $4 \mathrm{kG}$ for afmII would result in a separation of less than $0.2 \mathrm{~mm} \mathrm{~s}^{-1}$, within the width of the central Mössbauer peak. The small HFF for afmII results from the core and valence contributions having opposite sign and almost cancelling (table 2).

\section{Discussion}

The magnitude of TO mode splitting predicted here is related to the afmII magnetic structure, but any afm correlation will lead to the same effect. Above we have associated the major peak in the Raman spectrum with $\mathrm{TO}_{a}$, or afm nearest-neighbour correlations, and the satellite peak with $\mathrm{TO}_{b}$, or fm nearest-neighbour correlations. If the general character of a more complex magnetic state is indeed afm, a signal dominated by the afm peak $\left(\mathrm{TO}_{a}\right.$ in afmII) can be expected: in the case of a non-collinear spin state the satellite peak may even vanish, while the 
Table 2. Hyperfine field in bcc and afmII hcp iron under compression. Given are atomic volumes $V$, the corresponding pressure $P$ from the respective computational equation of state $[10,31]$, and the magnetic moment $M$ for the unit cell and inside the muffin tin sphere (in brackets) in Bohr magnetons. The total HFF $B$ and its contributions due to core $B_{\mathrm{c}}$ and valence electrons $B_{\mathrm{v}}$ follow. For afmII, $M$ and $B$ for the spin-up atoms are given. For comparison a previous calculation [32] for bcc iron is included. The calculations of the HFF were performed with the WIEN2k LAPW package. We find that including spin-orbit coupling has negligible effect on the HFF.

\begin{tabular}{llllllr}
\hline & $\begin{array}{l}V \\
\left(\mathrm{bohr}^{3}\right)\end{array}$ & $\begin{array}{l}P \\
(\mathrm{GPa})\end{array}$ & $\begin{array}{l}M \\
\left(\mu_{\mathrm{B}}\right)\end{array}$ & $\begin{array}{l}B \\
(\mathrm{kG})\end{array}$ & $\begin{array}{c}B_{\mathrm{c}} \\
(\mathrm{kG})\end{array}$ & $\begin{array}{l}B_{\mathrm{v}} \\
(\mathrm{kG})\end{array}$ \\
\hline bcc Fe & & & & & \\
\hline Exp & 79 & 0 & 2.13 & -339 & & \\
Reference [32] & 81 & & 2.46 & -316 & -280 & -36 \\
& 80 & -7 & $2.22(2.28)$ & -329 & -286 & -43 \\
& 75 & 6 & $2.14(2.18)$ & -309 & -279 & -31 \\
& 70 & 23 & $2.06(2.09)$ & -287 & -266 & -21 \\
& 65 & 48 & $1.90(1.92)$ & -261 & -249 & -12 \\
& 60 & 86 & $1.75(1.77)$ & -226 & -227 & 1 \\
\hline afmII Fe & & & & & & \\
\hline & 75 & -12 & $0(1.40)$ & 3 & -170 & 173 \\
& 70 & 2 & $0(1.10)$ & 4 & -132 & 136 \\
& 65 & 22 & $0(0.69)$ & 4 & -85 & 89 \\
& 60 & 56 & $0(0.20)$ & 2 & -31 & 33 \\
\hline
\end{tabular}

major peak still exhibits an afm signature through a shift in frequency relative to a hypothetical non-magnetic structure. Spin-phonon interactions have been found to have a strong effect on Raman scattering in a number of systems, including cupric oxide [36] and the copperruthenium oxide $\mathrm{RuSr}_{2} \mathrm{GdCu}_{2} \mathrm{O}_{8}$ [37]. The general character of the effect of spin-phonon interaction on the Raman spectra in these materials is consistent with the observations for hcp iron: broad, low amplitude, satellite peaks appear when the sample is below the Curie or Néel temperature.

Other experimental investigations of possible magnetic states in hcp iron have been inconclusive. Magnetism in hcp iron exists at over-expanded volumes in epitaxially grown multilayers on a ruthenium substrate [38]. Nuclear x-ray absorption experiments [39] show significant loss of moment across the phase transition from bcc to hcp iron, but cannot unambiguously be interpreted as the absence of moments in the high pressure polymorph: the change in absorption spectra is due to changes in the density of states as well as to spin-related satellites. It is worth noting that in the presence of an external field large internal magnetic fields develop in hcp iron [8], supporting our interpretation of hidden magnetic correlations in hep iron.

Long thought to be antithetical, superconductivity and magnetism are simultaneously observed in an increasing number of systems. Among these $\mathrm{ZrZn}_{2}$ is of particular interest: superconductivity and magnetism appear to be directly coupled, as evidenced by the loss of superconductivity and magnetism at the same pressure [40]. A similar connection between magnetism and superconductivity in hcp iron, which has only been observed recently [41], has now been investigated. While different in detail, two computational studies $[11,12]$ suggest that the rapid disappearance of superconductivity in hcp iron at about $30 \mathrm{GPa}$ is unconventional and is related to spin fluctuations.

In addition to the optical phonons, acoustic modes can also be influenced by spin-phonon interactions, as is evidenced by anomalous dispersion for the transverse acoustic phonon in 
fcc iron near the zone centre [3]. Indeed we find that, in addition to the bulk modulus, through the equation of state (figure 2 and table 1), the afmII ordering also influences the shear elastic properties of hcp iron: we find closer agreement for the shear modulus of afmII [42] with recent experimental estimates from ultrasonic measurements [43] and the phonon density of states [44].

\section{Conclusions}

Using a first-principles based magnetic tight-binding total energy model we have explored non-collinear magnetic structures in fcc and hcp iron. We find good qualitative agreement with previous first-principles results for spin density waves in the fcc structure. In hcp iron, in contrast, we find non-collinear solutions more stable over an orthorhombic collinear state (afmII) only at volumes close to and larger than the zero pressure volume. In the pressure stability range of hcp iron, above $13 \mathrm{GPa}$, however, the afmII structure is the lowest energy state found, predicting antiferromagnetic correlations up to $50 \mathrm{GPa}$.

Further investigating the collinear afmII structure, we have shown strong evidence for magnetic correlations in hcp iron in its stability field. We have calculated from first principles the effect of spin-phonon coupling on the zone centre TO mode frequency, and find good agreement with a recently observed unexpected splitting in the Raman frequencies of hcp iron under pressure. We have further calculated the hyperfine field of the afmII structure and obtain values of a few $\mathrm{kG}$ only, within the resolution limit of Mössbauer experiments. Combined with a considerably better agreement of structural and elastic properties between experiments on hcp iron and the afmII structure, as compared to non-magnetic calculations, this suggests that magnetic correlations do play an important role in understanding the physical properties of iron under pressure.

\section{Acknowledgments}

Thanks to P Blaha, B Fultz, A Goncharov, R Hemley, A Jephcoat, I Mazin, C McCammon, $\mathrm{S}$ Mukherjee, and V Struzhkin for helpful discussions. This work was supported by the US National Science Foundation under grants EAR-9980553 (to REC) and EAR-998002 (to LS), and the US Department of Energy ASCI/ASAP subcontract to Caltech, Grant DOE W7405-ENG-48 (to REC). Computations were performed on the CRAY SV1 at the Geophysical Laboratory, supported by NSF grant EAR-9975753 and the W M Keck Foundation.

\section{References}

[1] Bagno P, Jepsen O and Gunnarsson O 1989 Phys. Rev. B 401997

[2] Acet M et al 1994 Phys. Rev. B 496012

[3] Zaretsky J and Stassis C 1987 Phys. Rev. B 354500

[4] Cohen R E, Gramsch S, Steinle-Neumann G and Stixrude L 2002 Proc. Int. School of Physics 'Enrico Fermi' CXLVII ed R J Hemley et al (Washington, DC: IOS Press) p 215

[5] Millett J F C, Bourne N K and Rosenberg Z 1997 J. Appl. Phys. 812579

[6] Vocadlo L et al 2000 Phys. Earth Planet. Inter. 117123

[7] Cort G, Taylor R D and Willis J O 1982 J. Appl. Phys. 532064

[8] Taylor R D, Cort G and Willis J O 1982 J. Appl. Phys. 538199

[9] Nasu S et al 2002 J. Phys.: Condens. Matter 1411167

[10] Steinle-Neumann G, Stixrude L and Cohen R E 1999 Phys. Rev. B 60791

[11] Mazin I I, Papaconstantopoulos D A and Mehl M J 2002 Phys. Rev. B 65100511

[12] Jarlborg T 2002 Phys. Lett. A 300518

[13] Cohen R E and Mukherjee S 2004 Phys. Earth Planet. Inter. at press 
[14] Merkel S et al 2000 Science 2881626

[15] Goncharov A F et al 2002 Proc. Int. School of Physics 'Enrico Fermi' CXLVII ed R J Hemley et al (Washington, DC: IOS Press) p 297

[16] Jephcoat A P, Mao H K and Bell P M 1986 J. Geophys. Res. 914677

[17] Uhl M, Sandratskii L M and Kübler J 1994 Phys. Rev. B 50291

[18] Mryasov O, Gubanov V and Liechtenstein A 1992 Phys. Rev. B 4512330

[19] Tsunoda Y, Nishioka Y and Nicklow R 1991 J. Magn. Magn. Mater. 128133

[20] Mukherjee S and Cohen R E 2001 J. Comput.-Aided Mater. Des. 8107

[21] Diep H T 1992 Phys. Rev. B 452863

[22] Cohen R E, Mehl M J and Papaconstantopoulos D A 1994 Phys. Rev. B 5014694

[23] Pickett W E 1996 Korean Phys. Soc. 29 S70

[24] You M V and Heine V 1982 J. Phys. F: Met. Phys. 12177

[25] Freyss M, Stoeffler D and Dreysse H 1997 Phys. Rev. B 566047

[26] Mehl M J, Papaconstantopoulos D A, Mazin I I, Bacalis N and Pickett W E 2001 J. Appl. Phys. 896880

[27] Stoner E C 1938 Proc. R. Soc. A 169339

[28] Krasko G L 1987 Phys. Rev. B 368565

[29] Perdew J, Burke K and Ernzhofer M 1996 Phys. Rev. Lett. 773865

Perdew J, Burke K and Ernzhofer M 1996 Phys. Rev. Lett. 781396 (correction)

[30] Blügel S et al 1987 Phys. Rev. B 353271

[31] Stixrude L, Cohen R E and Singh D J 1994 Phys. Rev. B 506442

[32] Battocletti M, Ebert H and Akai H 1996 Phys. Rev. B 539776

[33] Sjöstedt E and Nordström L 2002 Phys. Rev. B 66014447

[34] Henley C L 1987 J. Appl. Phys. 613962

[35] Olijnyk H et al 2000 Phys. Rev. B 625508

[36] Chen X K, Irwin J C and Franck J P 1995 Phys. Rev. B 52 R13130

[37] Fainstein A et al 2000 Phys. Rev. B 6115468

[38] Saint-Lager M C et al 1995 Phys. Rev. B 512446

[39] Rueff J P et al 1999 Phys. Rev. B 6014510

[40] Pfleiderer C et al 2001 Nature $\mathbf{4 1 2} 58$

[41] Shimizu K et al 2001 Nature 412316

[42] Steinle-Neumann G, Stixrude L and Cohen R E 2002 EOS Trans. Am. Geophys. Union 83 MR62B-1069

[43] Mao H K et al 1998 Nature 396741

Mao H K et al 1998 Nature 399280 (correction)

[44] Mao H K et al 2001 Science 292914

[45] Mao H K, Wu Y, Chen L, Shu J and Jephcoat A P 1990 J. Geophys. Res. 9521737 